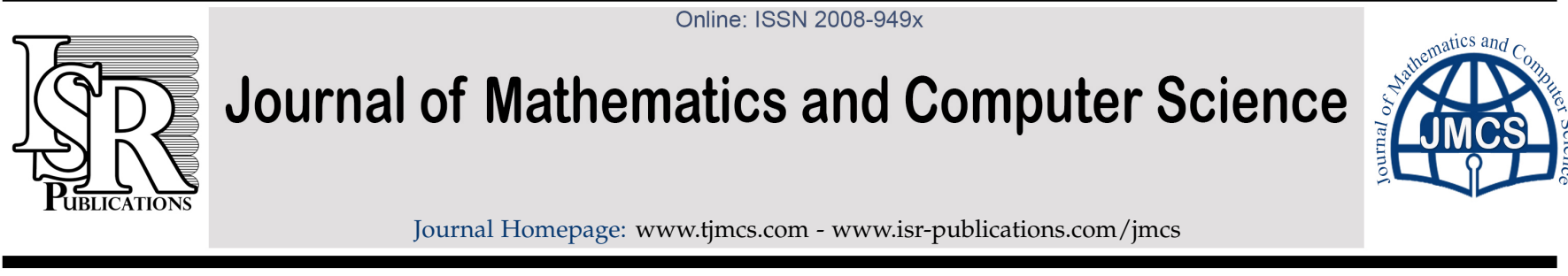

\title{
Symplectic properties research for finite element methods of Hamiltonian system
}

\author{
Qiong Tang ${ }^{\mathrm{a}, *}$, Yangfan Liu ${ }^{\mathrm{b}}$, Yujun Zheng ${ }^{\mathrm{c}}$, Hongping Cao ${ }^{\mathrm{d}}$ \\ ${ }^{a}$ Department of Information and Mathematics Science, Hunan University of Technology, Hunan, ZhuZhou, 412008, P. R. China. \\ ${ }^{b}$ College of materials science and engineering, Xiang Tan University, XiangTan 411105, Hunan, P. R. China. \\ ${ }^{c}$ Department of mathematics and Computational Science, Hunan University of Science and Engineering, YongZhou 425100, Hunan, \\ P. R. China. \\ ${ }^{d}$ College of Management, Shanghai University of Engineering Science, Shanghai, 201620, P. R. China.
}

\begin{abstract}
In this paper, we first apply properties of the wedge product and continuous finite element methods to prove that the linear, quadratic element methods are symplectic algorithms to the linear Hamiltonian systems, i.e., the symplectic condition $d p_{j+1} \wedge d q_{j+1}=d p_{j} \wedge d q_{j}$ is preserved exactly and the linear element method is an approximately symplectic integrator to nonlinear Hamiltonian systems, i.e., $d p_{j+1} \wedge d q_{j+1}=d p_{j} \wedge d q_{j}+O\left(h^{2}\right)$, as well as energy conservative.
\end{abstract}

Keywords: Hamiltonian systems, continuous finite element methods, energy conservative, wedge product, symplectic algorithm.

2010 MSC: 65P10, 65L60, 65L05.

(C)2018 All rights reserved.

\section{Introduction}

Hamiltonian system is a kind of important mechanical systems. It has a symplectic structure which has strong geometric properties of a dynamical system and maintain the total energy $\mathrm{H}(\mathrm{q}, \mathrm{p})$ which means the phase-space points $(q, p)$ are allowed on the constant energy hypersurface satisfying $H(q, p)=E$. It is natural to look for those discretization systems which preserve as much as possible the characteristic properties of the original continuous systems. Traditional algorithms, such as classical R-K method [4,5], Adams method etc. except some occasions are nonsymplectic, eventually lead to greatly distortions. Symplectic geometry is the mathematical foundation of Hamiltonian systems. Authors in [4-15] constructed the symplectic methods to solve the Hamiltonian system and got good results.

Consider the autonomous Hamiltonian systems:

$$
\frac{d p^{(i)}}{d t}=-\frac{\partial H}{\partial q^{(i)}}, \frac{d q^{(i)}}{d t}=\frac{\partial H}{\partial p^{(i)}}, i=1,2, \ldots, n,
$$

\footnotetext{
${ }^{*}$ Corresponding author

Email address: zzgxysx@163.com (Qiong Tang)
}

doi: $10.22436 /$ jmcs.018.03.07

Received: 2017-07-17 Revised: 2017-11-05 Accepted: 2018-04-24 
where $p=\left(p^{(1)}, p^{(2)}, \ldots, p^{(n)}\right)^{\top}, q=\left(q^{(1)}, q^{(2)}, \ldots, q^{(n)}\right)^{\top}$, 'T' is matrix transpose. In applications to mechanics, $q$ represents the space coordinates, $p$ the momenta, and $H$ is the energy of systems.

Let $z=(p, q)^{\top}, \frac{\partial H}{\partial z}=H_{z} \in R^{2 n}$. Then (1.1) can be written as

$$
\frac{\mathrm{d} z}{\mathrm{dt}}=\mathrm{J}^{-1} \frac{\partial \mathrm{H}}{\partial z}, \quad J=\left[\begin{array}{cc}
0 & \mathrm{I}_{\mathrm{n}} \\
-\mathrm{I}_{\mathrm{n}} & 0
\end{array}\right]
$$

$\mathrm{I}_{\mathrm{n}}$ is the $\mathrm{n} \times \mathrm{n}$ identity matrix, $\mathrm{J}^{\mathrm{T}}=\mathrm{J}^{-1}=-\mathrm{J}$.

Let $\Psi$ be a diffeomorphism of $\mathrm{R}^{2 \mathrm{n}}$ :

$$
z=\left(\begin{array}{c}
p \\
q
\end{array}\right) \rightarrow \Psi(z)=\left[\begin{array}{c}
\Psi^{(1)}(z) \\
\cdot \\
\cdot \\
\Psi^{(2 n)}(z)
\end{array}\right]=\left[\begin{array}{c}
\hat{p}(p, q) \\
\hat{q}(p, q)
\end{array}\right]
$$

Definition 1.1. A smooth map $\Psi$ on the phase space $R^{2 n}$ is called a symplectic map or canonical map if its Jacobi $\Psi_{z}(z)$ satisfies:

$$
\left[\Psi_{z}(z)\right]^{\mathrm{T}} \mathrm{J} \Psi_{z}(z)=\mathrm{J}
$$

for all $z$ in the domain of definition of $\Psi$ [4].

Equation (1.1) or (1.2) be defined in phase space $R^{2 n}$ with a standard symplectic structure given by the non-singular anti-symmetric closed differential 2-form

$$
\omega=\sum_{i=1}^{n} d z^{(i)} \wedge d z^{(n+i)}=\sum_{i=1}^{n} d p^{(i)} \wedge d q^{(i)}=d p \wedge d q .
$$

$\Psi$ is called a symplectic transformation if $\Psi$ preserves the 2-form $\omega$

$$
\sum_{i=1}^{n} d \hat{p}^{(i)} \wedge d \hat{q}^{(i)}=\sum_{i=1}^{n} d p^{(i)} \wedge d q^{(i)} .
$$

This is equivalent to the condition that [4]

$$
\left(\frac{\partial \Psi}{\partial z}\right)^{\top} \mathrm{J}\left(\frac{\partial \Psi}{\partial z}\right)=\mathrm{J}
$$

Thus conservation of symplecticness, under a transformation $\hat{p}=\Phi^{1}(p, q), \hat{q}=\Phi^{2}(p, q)$ reduces to the statement

$$
\mathrm{d} \hat{\mathrm{p}} \wedge \mathrm{d} \hat{\mathrm{q}}=\mathrm{dp} \wedge \mathrm{dq},
$$

where $d \hat{p}=\Phi_{p}^{1}(p, q) d p+\Phi_{q}^{1}(p, q) d q, d \hat{q}=\Phi_{p}^{2}(p, q) d p+\Phi_{q}^{2}(p, q) d q$.

Definition 1.2. A numerical method is symplectic integrator if the symplecticness condition $d p_{j+1} \wedge$ $\mathrm{dq}_{\mathrm{j}+1}=\mathrm{dp}_{\mathrm{j}} \wedge \mathrm{dq}_{\mathrm{j}}$ is preserved exactly [11].

Wedge product is a differential 2-form, with the following properties:

1. skew-symmetry:

$$
\mathrm{da} \wedge \mathrm{db}=-\mathrm{db} \wedge \mathrm{da}
$$

2. bilinearity:

$$
\text { for } \alpha, \beta \in R, \quad d a \wedge(\alpha d b+\beta d c)=\alpha d a \wedge d b+\beta d a \wedge d c
$$


3. rule of matrix multiplication (as a consequence of Property 2 and the definition)

$$
\mathrm{da} \wedge(\mathrm{Adb})=\left(A^{\top} \mathrm{da}\right) \wedge d b \text {, for any } n \times n \text { matrix } A \text {; }
$$

4.

if $A$ is a symmetric matrix, then $\mathrm{da} \wedge(\mathrm{Ada})=0$,

where $d a, d b$, and $d c$ are $n$-vectors of differential one-forms on $R^{n}$.

The definition $\Psi_{z}(z)^{\top} J \Psi_{z}(z)=\mathrm{J}$ is not always the most convenient approach to check the symplecticness of a given map $\Psi$. The wedge product notation can be combined with implicit differentiation, which makes it a powerful tool to verify symplecticness of an implicitly given transformation $\Psi$. Leimkuhler and Reich in [11] utilized the wedge product and composition method proving that Euler-A, Euler-B, implicit midpoint method for the general Hamiltonian and the second-order Stormer-Verlet methods for the special case of a separable Hamiltonian are canonically symplectic. Sanz-Serna [14], Sanz-Serna and Calvo [15], Lasagni [10], and Suris [17] utilized the wedge product and tensor product to prove the conditions of the parameters $a_{i j}$ and $b_{i}$ are $b_{i} a_{i j}+b_{j} a_{j i}-b_{i} b_{j}=0, i, j=1, \ldots, s$ for a s-stage symplectic Runge-Kutta method. So the wedge product is also an important method to study symplectic geometry algorithm of Hamiltonian system.

Symplectic algorithm is a difference method that preserves the structure of the system. However, most numerical methods can't maintain the two properties: symplectic and energy conservation simultaneously in general according to Ge-Marsde theorem [6]. Symplectic algorithm can preserves symplectic properties, but only obtain approximate energy conservation for nonlinear Hamiltonian system.

Many scholars pointed out that the energy conservation is more important at certain times, see $[8,16]$. So we turn to the finite element method (FEM). It is founded that the continuous FEM always preserves the energy [18], thus we need only to discuss symplectic properties. In this paper, we apply continuous FEM and properties of the wedge product to prove that the linear, quadratic element methods are symplectic algorithms to the linear Hamiltonian systems, i.e.the symplectic condition $d p_{j+1} \wedge d q_{j+1}=d p_{j} \wedge d q_{j}$ is preserved exactly and the linear element method is an approximately symplectic integrator to nonlinear Hamiltonian systems, i.e., $d p_{j+1} \wedge d q_{j+1}=d p_{j} \wedge d q_{j}+O\left(h^{2}\right)$.

\section{Continuous FEM for Hamiltonian system}

Consider the first-order ordinary differential equation with initial value in the interval $\mathrm{I}=[0, \mathrm{~T}]$

$$
\frac{d u}{d t}=u^{\prime}=f(t, u), u(0)=u_{0} \text {. }
$$

Set $\triangle^{h}: t_{0}=0<t_{1}<t_{2}<\cdots<t_{N}=T$ as partition of $I$, with interval $I_{j}=\left(t_{j}, t_{j+1}\right), h_{j}=t_{j+1}-t_{j}$. Define the $m$-th degree continuous finite element space $[2,3]$

$$
S^{h}=\left\{w|w \in C(I), w|_{I_{j}} \in P_{m}\right\},
$$

where $P_{m}$ is a $m$-th degree polynomial. Each $m$-degree polynomial in interval $I_{j}$ has $m+1$ parameters, but only $m$ freedoms, as its starting value at point $t_{j}$ is given. Define $m$-th degree continuous finite element $\mathrm{U} \in \mathrm{S}^{\mathrm{h}}$ satisfying

$$
\int_{\mathrm{I}_{\mathrm{j}}}\left(\mathrm{U}^{\prime}-\mathrm{f}(\mathrm{t}, \mathrm{U})\right) v \mathrm{dt}=0, \quad v \in \mathrm{P}_{\mathrm{m}-1}, \mathrm{U}(0)=\mathrm{u}_{0},
$$

i.e., it is orthogonal to arbitrary $\mathrm{P}_{\mathrm{m}-1}$ in $\mathrm{I}_{\mathrm{j}}$. Taking $v \in \mathrm{S}^{\mathrm{h}}$, its derivate $v^{\prime} \in \mathrm{P}_{\mathrm{m}-1}$. In practical computation, take $v=\left(t-t_{j}\right)^{i}, i=0,1,2, \ldots, m-1$.

Lemma 2.1 ([3]). The $m$-th degree continuous finite element of ordinary differential equation has superconvergence in nodes $t_{j}$

$$
(u-u)\left(t_{j}\right)=O\left(h^{2 m}\right)\|u\|_{m+1, \infty} .
$$


In (1.2), the $m$-th degree continuous finite element $Z=\left[\begin{array}{l}P \\ Q\end{array}\right]$ of $z$ satisfies orthogonal relation:

$$
\int_{\mathrm{I}_{j}}\left(\mathrm{Z}^{\prime}-\mathrm{J}^{-1} \mathrm{H}_{z}\right) v^{\prime} \mathrm{dt}=0, \mathrm{Z}(0)=z_{0}
$$

Taking $v=\left[\begin{array}{l}\mathrm{Q} \\ \mathrm{P}\end{array}\right]$, we obtain:

$$
\int_{I_{j}}\left(P^{\prime}+H_{q}(P, Q)\right) Q^{\prime} d t=0, P(0)=p_{0}, \quad \int_{I_{j}}\left(Q^{\prime}-H_{p}(P, Q)\right) P^{\prime} d t=0, Q(0)=q_{0} .
$$

It follows from the above two equations that:

$$
\int_{I_{j}}\left(H_{p} P^{\prime}+H_{q} Q^{\prime}\right) d t=\int_{I_{j}} \frac{d}{d t} H(P, Q) d t=0
$$

Hence, in each nodes $t_{j}$, we prove that:

$$
\mathrm{H}\left(\mathrm{P}\left(\mathrm{t}_{\mathrm{j}}\right), \mathrm{Q}\left(\mathrm{t}_{\mathrm{j}}\right)\right)=\mathrm{H}\left(\mathrm{P}\left(\mathrm{t}_{\mathbf{j}-1}\right), \mathrm{Q}\left(\mathrm{t}_{\mathrm{j}-1}\right)\right)=\cdots=\mathrm{H}(\mathrm{P}(0), \mathrm{Q}(0))=\mathrm{H}\left(\mathrm{p}_{0}, \mathrm{q}_{0}\right) .
$$

So we can prove that

Lemma 2.2 ([18]). Applying arbitrary degree continuous finite element to solve Hamilton equation, it maintains energy conservation, i.e., $\mathrm{H}\left(\mathrm{P}\left(\mathrm{t}_{\mathbf{j}}\right), \mathrm{Q}\left(\mathrm{t}_{\mathbf{j}}\right)\right)=\mathrm{H}\left(\mathrm{p}_{0}, \mathrm{q}_{0}\right)$.

\section{The wedge product notation applied in the linear element methods}

Consider the linear Hamiltonian system,

$$
\frac{\mathrm{d} z}{\mathrm{dt}}=\mathrm{J}^{-1} \mathrm{Lz}, \mathrm{Z}(0)=z_{0}
$$

where $H(z)=\frac{1}{2} z^{\top} L z, L^{\top}=L=\left(\begin{array}{cc}A & C \\ C^{\top} & B\end{array}\right)$, and $A, B$ are $n \times n$ symmetric matrices. Thus the canonical equation as follows

$$
\frac{d p}{d t}=-C^{\top} p-B q, \frac{d q}{d t}=A p+C q .
$$

Utilizing linear FEM in the interval $I_{j}=\left[t_{j}, t_{j+1}\right]$ :

$$
\int_{I_{j}} \frac{d P}{d t} * 1 d t=-\int_{I_{j}}\left(C^{T} P+B Q\right) * 1 d t, \quad \int_{I_{j}} \frac{d Q}{d t} * 1 d t=\int_{I_{j}}(A P+C Q) * 1 d t,
$$

where the linear element of $p$ is $P=\frac{t-t_{j+1}}{t_{j}-t_{j+1}} P_{j}+\frac{t-t_{j}}{t_{j+1}-t_{j}} P_{j+1}$ and $q$ is $Q=\frac{t-t_{j+1}}{t_{j}-t_{j+1}} Q_{j}+\frac{t-t_{j}}{t_{j+1}-t_{j}} Q_{j+1}$.

Integral (3.1)

$$
\begin{aligned}
P_{j+1}-P_{j} & =-\frac{h_{j}}{2}\left(C^{\top}\left(P_{j}+P_{j+1}\right)+B\left(Q_{j}+Q_{j+1}\right)\right), \\
Q_{j+1}-Q_{j} & =\frac{h_{j}}{2}\left(A\left(P_{j}+P_{j+1}\right)+C\left(Q_{j}+Q_{j+1}\right)\right), j=0,1, \ldots, N-1 .
\end{aligned}
$$

By taking differential of (3.2) we can write

$$
\begin{aligned}
d P_{j+1}-d P_{j} & =-\frac{h_{j}}{2}\left(C^{\top}\left(d P_{j}+d P_{j+1}\right)+B\left(d Q_{j}+d Q_{j+1}\right)\right), \\
d Q_{j+1}-d Q_{j} & =\frac{h_{j}}{2}\left(A\left(d P_{j}+d P_{j+1}\right)+C\left(d Q_{j}+d Q_{j+1}\right)\right) .
\end{aligned}
$$


From (3.3), we can obtain

$$
\begin{aligned}
& \left(I+\frac{h_{j} C^{\top}}{2}\right) d P_{j+1}+\frac{h_{j} B}{2} d Q_{j+1}=\left(I-\frac{h_{j} C^{\top}}{2}\right) d P_{j}-\frac{h_{j} B}{2} d Q_{j}, \\
& -\frac{h_{j} A}{2} d P_{j+1}+\left(I-\frac{h_{j} C}{2}\right) d Q_{j+1}=\frac{h_{j} A}{2} d P_{j}+\left(I+\frac{h_{j} C}{2}\right) d Q_{j},
\end{aligned}
$$

by taking wedge products with $d_{Q_{j+1}}$ and $d P_{j+1}$, respectively. With symmetric properties of $A$ and $B$ and according to (1.5)

thus

$$
\frac{h_{j} B}{2} d Q_{j+1} \wedge d Q_{j+1}=0,-\frac{h_{j} A}{2} d_{j+1} \wedge d P_{j+1}=0,
$$

$$
\begin{gathered}
\left(I+\frac{h_{j} C^{\top}}{2}\right) d P_{j+1} \wedge d Q_{j+1}=\left(I-\frac{h_{j} C^{\top}}{2}\right) d P_{j} \wedge d Q_{j+1}-\frac{h_{j} B}{2} d Q_{j} \wedge d Q_{j+1}, \\
\left(I-\frac{h_{j} C}{2}\right) d Q_{j+1} \wedge d P_{j+1}=\frac{h_{j} A}{2} d P_{j} \wedge d P_{j+1}+\left(I+\frac{h_{j} C}{2}\right) d Q_{j} \wedge d P_{j+1} .
\end{gathered}
$$

By subtracting the above equations we have

$$
\begin{aligned}
2 \mathrm{dP}_{j+1} \wedge d Q_{j+1}= & \left(I-\frac{h_{j} C^{T}}{2}\right) d P_{j} \wedge d Q_{j+1}-\frac{h_{j} B}{2} d Q_{j} \wedge d Q_{j+1} \\
& -\frac{h_{j} A}{2} d P_{j} \wedge d P_{j+1}-\left(I+\frac{h_{j} C}{2}\right) d Q_{j} \wedge d P_{j+1} .
\end{aligned}
$$

Similarly, If we take the wedge product of (3.4) with $d Q_{j}$ and (3.5) with $\mathrm{dP}_{j}$, then subtract

$$
\begin{aligned}
2 \mathrm{dP}_{j} \wedge d Q_{j}= & \left(I+\frac{h_{j} C^{\top}}{2}\right) d P_{j+1} \wedge d Q_{j}+\frac{h_{j} B}{2} d Q_{j+1} \wedge d Q_{j} \\
& +\frac{h_{j} A}{2} d P_{j+1} \wedge d P_{j}-\left(I-\frac{h_{j} C}{2}\right) d Q_{j+1} \wedge d P_{j} .
\end{aligned}
$$

Based on (1.3), (1.4), (3.6), and (3.7), we prove the following equation

$$
\mathrm{dP}_{\mathrm{j}+1} \wedge \mathrm{dQ}_{\mathrm{j}+1}=\mathrm{dP}_{\mathbf{j}} \wedge \mathrm{dQ} \mathrm{Q}_{\mathbf{j}} .
$$

Theorem 3.1. The linear finite element method for the linear Hamiltonian systems is a symplectic algorithm, i.e., the symplectic condition $\mathrm{dp}_{j+1} \wedge \mathrm{dq}_{j+1}=\mathrm{dp}_{\mathrm{j}} \wedge \mathrm{dq}_{\mathrm{j}}$ is preserved exactly.

\section{The wedge product notation applied in the quadratic element methods}

Utilizing quadratic element methods in the interval $I_{j}=\left[t_{j}, t_{j+1}\right]$ :

$$
\begin{aligned}
\int_{I_{j}} \frac{d P}{d t} * 1 d t & =-\int_{I_{j}}\left(C^{T} P+B Q\right) * 1 d t, \\
\int_{I_{j}} \frac{d Q}{d t} * 1 d t & =\int_{I_{j}}(A P+C Q) * 1 d t \\
\int_{I_{j}} \frac{d P}{d t} *\left(t-t_{j}\right) d t & =-\int_{I_{j}}\left(C^{\top} P+B Q\right) *\left(t-t_{j}\right) d t \\
\int_{I_{j}} \frac{d Q}{d t} *\left(t-t_{j}\right) d t & =\int_{I_{j}}(A P+C Q) *\left(t-t_{j}\right) d t,
\end{aligned}
$$

where the quadratic element of $p$ is

$$
P=\frac{\left(t-t_{j+1}\right)\left(t-t_{j+\frac{1}{2}}\right)}{\left(t_{j}-t_{j+1}\right)\left(t_{j}-t_{j+\frac{1}{2}}\right)} P_{j}+\frac{\left(t-t_{j+1}\right)\left(t-t_{j}\right)}{\left(t_{j+\frac{1}{2}}-t_{j+1}\right)\left(t_{j+\frac{1}{2}}-t_{j}\right)} P_{j+\frac{1}{2}}+\frac{\left(t-t_{j+\frac{1}{2}}\right)\left(t-t_{j}\right)}{\left(t_{j+1}-t_{j+\frac{1}{2}}\right)\left(t_{j+1}-t_{j}\right)} P_{j+1}
$$

and $q$ is 


$$
Q=\frac{\left(t-t_{j+1}\right)\left(t-t_{j+\frac{1}{2}}\right)}{\left(t_{j}-t_{j+1}\right)\left(t_{j}-t_{j+\frac{1}{2}}\right)} Q_{j}+\frac{\left(t-t_{j+1}\right)\left(t-t_{j}\right)}{\left(t_{j+\frac{1}{2}}-t_{j+1}\right)\left(t_{j+\frac{1}{2}}-t_{j}\right)} Q_{j+\frac{1}{2}}+\frac{\left(t-t_{j+\frac{1}{2}}\right)\left(t-t_{j}\right)}{\left(t_{j+1}-t_{j+\frac{1}{2}}\right)\left(t_{j+1}-t_{j}\right)} Q_{j+1}
$$

in $I_{j}$.

Integral (4.1)

$$
\begin{aligned}
P_{j+1}-P_{j} & =-\frac{h_{j}}{2}\left(C^{\top}\left(\frac{P_{j}}{3}+\frac{4 P_{j+1 / 2}}{3}+\frac{P_{j+1}}{3}\right)+B\left(\frac{Q_{j}}{3}+\frac{4 Q_{j+1 / 2}}{3}+\frac{Q_{j+1}}{3}\right)\right), \\
Q_{j+1}-Q_{j} & =\frac{h_{j}}{2}\left(A\left(\frac{P_{j}}{3}+\frac{4 P_{j+1 / 2}}{3}+\frac{P_{j+1}}{3}\right)+C\left(\frac{Q_{j}}{3}+\frac{4 Q_{j+1 / 2}}{3}+\frac{Q_{j+1}}{3}\right)\right), \\
\frac{5 P_{j+1}}{6}-\frac{P_{j}}{6}-\frac{2 P_{j+1 / 2}}{3} & =-\frac{h}{4}\left(C^{\top}\left(\frac{4 P_{j+1 / 2}}{3}+\frac{2 P_{j+1}}{3}\right)+B\left(\frac{4 Q_{j+1 / 2}}{3}+\frac{2 Q_{j+1}}{3}\right)\right), \\
\frac{5 Q_{j+1}}{6}-\frac{Q_{j}}{6}-\frac{2 Q_{j+1 / 2}}{3} & =\frac{h}{4}\left(A\left(\frac{4 P_{j+1 / 2}}{3}+\frac{2 P_{j+1}}{3}\right)+C\left(\frac{4 Q_{j+1 / 2}}{3}+\frac{2 Q_{j+1}}{3}\right)\right) .
\end{aligned}
$$

By taking differential of (4.2) we have

$$
\begin{gathered}
\frac{2 h_{j} C^{\top}}{3} d P_{j+1 / 2}+\left(I+\frac{h_{j} C^{\top}}{6}\right) d P_{j+1}+\frac{2 h_{j} B}{3} d Q_{j+1 / 2}+\frac{h_{j} B}{6} d Q_{j+1}=\left(I-\frac{h_{j} C^{\top}}{6}\right) d P_{j}-\frac{h_{j} B}{6} d Q_{j}, \\
-\frac{2 h_{j} A}{3} d P_{j+1 / 2}-\frac{h_{j} A}{6} d P_{j+1}-\frac{2 h_{j} C}{3} d Q_{j+1 / 2}+\left(I-\frac{h_{j} C}{6}\right) d Q_{j+1}=\frac{h_{j} A}{6} d P_{j}+\left(I+\frac{h_{j} C}{6}\right) d Q_{j}, \\
\left(-\frac{2 I}{3}+\frac{h_{j} C^{\top}}{3}\right) d P_{j+1 / 2}+\left(\frac{5 I}{6}+\frac{h_{j} C^{\top}}{6}\right) d P_{j+1}+\frac{h_{j} B}{3} d Q_{j+1 / 2}+\frac{h_{j} B}{6} d Q_{j+1}=\frac{d P_{j}}{6}, \\
-\frac{h_{j} A}{3} d P_{j+1 / 2}-\frac{h_{j} A}{6} d P_{j+1}+\left(-\frac{2 I}{3}-\frac{h_{j} C}{3}\right) d Q_{j+1 / 2}+\left(\frac{5 I}{6}-\frac{h_{j} C}{6}\right) d Q_{j+1}=\frac{d Q_{j}}{6} .
\end{gathered}
$$

By taking wedge product of (4.3) with $\mathrm{dQ}_{\mathbf{j}+1}$, and (4.4) with $\mathrm{dP}_{\mathbf{j}+1}$, then subtract

$$
\begin{aligned}
2 d P_{j+1} \wedge d Q_{j+1}= & -\frac{2 h_{j} C^{T}}{3} d P_{j+1 / 2} \wedge d Q_{j+1}-\frac{2 h_{j} B}{3} d Q_{j+1 / 2} \wedge d Q_{j+1}-\frac{2 h_{j} A}{3} d P_{j+1 / 2} \wedge d P_{j+1} \\
& -\frac{2 h_{j} C}{3} d Q_{j+1 / 2} \wedge d P_{j+1}+\left(I-\frac{h_{j} C^{\top}}{6}\right) d P_{j} \wedge d Q_{j+1}-\frac{h_{j} B}{6} d Q_{j} \wedge d Q_{j+1} \\
& -\frac{h_{j} A}{6} d P_{j} \wedge d P_{j+1}-\left(I+\frac{h_{j} C}{6}\right) d Q_{j} \wedge d P_{j+1} .
\end{aligned}
$$

Similarly, we take the wedge product of (4.3) with $\mathrm{dQ}_{j}$ and (4.4) with $\mathrm{dP}_{j}$, then subtract

$$
\begin{aligned}
2 \mathrm{dP}_{j} \wedge d Q_{j}= & \frac{2 h_{j} C^{\top}}{3} d P_{j+1 / 2} \wedge d Q_{j}+\frac{2 h_{j} B}{3} d Q_{j+1 / 2} \wedge d Q_{j}+\frac{2 h_{j} A}{3} d P_{j+1 / 2} \wedge d P_{j} \\
& +\frac{2 h_{j} C}{3} d Q_{j+1 / 2} \wedge d P_{j}+\left(I+\frac{h_{j} C^{\top}}{6}\right) d P_{j+1} \wedge d Q_{j}+\frac{h_{j} B}{6} d Q_{j+1} \wedge d Q_{j} \\
& +\frac{h_{j} A}{6} d P_{j+1} \wedge d P_{j}-\left(I-\frac{h_{j} C}{6}\right) d Q_{j+1} \wedge d P_{j} .
\end{aligned}
$$

By subtracting (4.8) from (4.7) we have

$$
\begin{aligned}
d P_{j} \wedge d Q_{j}-d P_{j+1} \wedge d Q_{j+1}= & \frac{h_{j} C^{\top}}{3} d P_{j+1 / 2} \wedge d Q_{j}+\frac{h_{j} B}{3} d Q_{j+1 / 2} \wedge d Q_{j} \\
& +\frac{h_{j} A}{3} d P_{j+1 / 2} \wedge d P_{j}+\frac{h_{j} C}{3} d Q_{j+1 / 2} \wedge d P_{j}+\frac{h_{j} C^{\top}}{3} d P_{j+1 / 2} \wedge d Q_{j+1} \\
& +\frac{h_{j} B}{3} d Q_{j+1 / 2} \wedge d Q_{j+1}+\frac{h_{j} A}{3} d P_{j+1 / 2} \wedge d P_{j+1} \\
& +\frac{h_{j} C}{3} d Q_{j+1 / 2} \wedge d P_{j+1} .
\end{aligned}
$$


By taking wedge product of (4.5) with $\mathrm{dQ}_{j},(4.6)$ with $d P_{j}$, then subtract:

$$
\begin{aligned}
\frac{1}{3} d P_{j} \wedge d Q_{j}= & \left(-\frac{2 I}{3}+\frac{h_{j} C^{T}}{3}\right) d P_{j+1 / 2} \wedge d Q_{j}+\left(\frac{5 I}{6}+\frac{h_{j} C^{T}}{6}\right) d P_{j+1} \wedge d Q_{j} \\
& +\frac{h_{j} B}{3} d Q_{j+1 / 2} \wedge d Q_{j}+\frac{h_{j} B}{6} d Q_{j+1} \wedge d Q_{j}+\frac{h_{j} A}{3} d P_{j+1 / 2} \wedge d P_{j}+\frac{h_{j} A}{6} d P_{j+1} \wedge d P_{j} \\
& +\left(\frac{2 I}{3}+\frac{h_{j} C}{3}\right) d Q_{j+1 / 2} \wedge d P_{j}-\left(\frac{5 I}{6}-\frac{h_{j} C}{6}\right) d Q_{j+1} \wedge d P_{j} .
\end{aligned}
$$

By subtracting (4.8) from (4.10) we have

$$
\begin{aligned}
\frac{5}{3} \mathrm{dP}_{j} \wedge d Q_{j}= & \left(\frac{2 I}{3}+\frac{h_{j} C^{\top}}{3}\right) d P_{j+1 / 2} \wedge d Q_{j}+\frac{I}{6} d P_{j+1} \wedge d Q_{j}+\frac{h_{j} B}{3} d Q_{j+1 / 2} \wedge d Q_{j} \\
& +\frac{h_{j} A}{3} d P_{j+1 / 2} \wedge d P_{j}+\left(-\frac{2 I}{3}+\frac{h_{j} C}{3}\right) d Q_{j+1 / 2} \wedge d P_{j}-\frac{I}{6} d Q_{j+1} \wedge d P_{j} .
\end{aligned}
$$

By taking wedge product of (4.5) with $\mathrm{dQ}_{j+1}$, (4.6) with $\mathrm{dP}_{j+1}$, then subtract, we have

$$
\begin{aligned}
-\frac{5}{3} d P_{j+1} \wedge d Q_{j+1}= & \left(-\frac{2 I}{3}+\frac{h_{j} C^{\top}}{3}\right) d P_{j+1 / 2} \wedge d Q_{j+1}+\left(\frac{2 I}{3}+\frac{h_{j} C}{3}\right) d Q_{j+1 / 2} \wedge d P_{j+1} \\
& +\frac{h_{j} B}{3} d Q_{j+1 / 2} \wedge d Q_{j+1}+\frac{h_{j} A}{3} d P_{j+1 / 2} \wedge d P_{j+1} \\
& -\frac{I}{6} d P_{j} \wedge d Q_{j+1}+\frac{I}{6} d Q_{j} \wedge d P_{j+1} .
\end{aligned}
$$

By adding the above two equations (4.11) and (4.12):

$$
\begin{aligned}
\frac{5}{3} d P_{j} & \wedge d Q_{j}-\frac{5}{3} d P_{j+1} \wedge d Q_{j+1} \\
= & \left(\frac{2 I}{3}+\frac{h_{j} C^{T}}{3}\right) d P_{j+1 / 2} \wedge d Q_{j}+\frac{h_{j} B}{3} d Q_{j+1 / 2} \wedge d Q_{j} \\
& +\frac{h_{j} A}{3} d P_{j+1 / 2} \wedge d P_{j}+\left(-\frac{2 I}{3}+\frac{h_{j} C}{3}\right) d Q_{j+1 / 2} \wedge d P_{j}+\left(-\frac{2 I}{3}+\frac{h_{j} C^{T}}{3}\right) d P_{j+1 / 2} \wedge d Q_{j+1} \\
& +\left(\frac{2 I}{3}+\frac{h_{j} C}{3}\right) d Q_{j+1 / 2} \wedge d P_{j+1}+\frac{h_{j} B}{3} d Q_{j+1 / 2} \wedge d Q_{j+1}+\frac{h_{j} A}{3} d P_{j+1 / 2} \wedge d P_{j+1} .
\end{aligned}
$$

Utilizing (4.9), we can prove that:

$$
d P_{j} \wedge d Q_{j}-d P_{j+1} \wedge d Q_{j+1}=d P_{j+\frac{1}{2}} \wedge d Q_{j}-d Q_{j+\frac{1}{2}} \wedge d P_{j}-d P_{j+\frac{1}{2}} \wedge d Q_{j+1}+d Q_{j+\frac{1}{2}} \wedge d P_{j+1} .
$$

By taking wedge product of (4.3) with $\mathrm{dQ}_{j+1 / 2},(4.4)$ with $\mathrm{dP}_{j+1 / 2}$, then subtract.

$$
\begin{aligned}
& -d P_{j+1 / 2} \wedge d Q_{j}+d Q_{j+\frac{1}{2}} \wedge d P_{j}+d P_{j+\frac{1}{2}} \wedge d Q_{j+1}-d Q_{j+\frac{1}{2}} \wedge d P_{j+1}= \\
& \frac{h_{j} C^{T}}{6} d P_{j+\frac{1}{2}} \wedge d Q_{j+1}+\frac{h_{j} B}{6} d Q_{j+\frac{1}{2}} \wedge d Q_{j+1}+\frac{h_{j} A}{6} d P_{j+\frac{1}{2}} \wedge d P_{j+1}+\frac{h_{j} C}{6} d Q_{j+\frac{1}{2}} \wedge d P_{j+1} \\
& \frac{h_{j} C^{T}}{6} d P_{j+\frac{1}{2}} \wedge d Q_{j}+\frac{h_{j} B}{6} d Q_{j+\frac{1}{2}} \wedge d Q_{j}+\frac{h_{j} A}{6} d P_{j+\frac{1}{2}} \wedge d P_{j}+\frac{h_{j} C}{6} d Q_{j+\frac{1}{2}} \wedge d P_{j} . \\
& -d P_{j+1 / 2} \wedge d Q_{j}+d Q_{j+\frac{1}{2}} \wedge d P_{j}+d P_{j+\frac{1}{2}} \wedge d Q_{j+1}-d Q_{j+\frac{1}{2}} \wedge d P_{j+1} \\
& =\frac{h_{j} C^{T}}{6} d P_{j+\frac{1}{2}} \wedge d Q_{j+1}+\frac{h_{j} B}{6} d Q_{j+\frac{1}{2}} \wedge d Q_{j+1}+\frac{h_{j} A}{6} d P_{j+\frac{1}{2}} \wedge d P_{j+1}+\frac{h_{j} C}{6} d Q_{j+\frac{1}{2}} \wedge d P_{j+1} \\
& \quad+\frac{h_{j} C^{T}}{6} d P_{j+\frac{1}{2}} \wedge d Q_{j}+\frac{h_{j} B}{6} d Q_{j+\frac{1}{2}} \wedge d Q_{j}+\frac{h_{j} A}{6} d P_{j+\frac{1}{2}} \wedge d P_{j}+\frac{h_{j} C}{6} d Q_{j+\frac{1}{2}} \wedge d P_{j} .
\end{aligned}
$$


Combining (4.9) and (4.13), we obtain:

$$
-d P_{j} \wedge d Q_{j}+d P_{j+1} \wedge d Q_{j+1}=\frac{d P_{j} \wedge d Q_{j}}{2}-\frac{d P_{j+1} \wedge d Q_{j+1}}{2},
$$

i.e., $d P_{j+1} \wedge d Q_{j+1}=d P_{j} \wedge d Q_{j}$. We can prove the following theorem.

Theorem 4.1. The quadratic finite element method for the linear Hamiltonian systems is a symplectic algorithm, i.e., the symplectic condition $\mathrm{dp}_{j+1} \wedge \mathrm{dq}_{j+1}=\mathrm{dp}_{\mathrm{j}} \wedge \mathrm{dq}_{\mathrm{j}}$ is preserved exactly.

\section{The wedge product notation applied in the nonlinear Hamiltonian system}

Consider the general canonical system $\mathrm{H}(\mathrm{q}, \mathrm{p})$

$$
\frac{d p}{d t}=-\frac{\partial H}{\partial q}, \frac{d q}{d t}=\frac{\partial H}{\partial p} .
$$

In interval $I_{j}=\left[t_{j}, t_{j+1}\right]$ :

$$
\int_{I_{j}} \frac{d P}{d t} * 1 d t=-\int_{I_{j}} \frac{\partial H}{\partial q} * 1 d t, \quad \int_{I_{j}} \frac{d Q}{d t} * 1 d t=\int_{I_{j}} \frac{\partial H}{\partial p} * 1 d t, j=0,1, \ldots, N-1 .
$$

Integral (5.1)

$$
\begin{aligned}
P_{j+1}-P_{j} & =-\left.\frac{h_{j}}{2} \int_{-1}^{1} \frac{\partial H}{\partial q}\right|_{p=\frac{1-x}{2}} P_{j}+\frac{1+x}{2} P_{j+1}, q=\frac{1-x}{2} Q_{j}+\frac{1+x}{2} Q_{j+1} d x, \\
Q_{j+1}-Q_{j} & =\left.\frac{h_{j}}{2} \int_{-1}^{1} \frac{\partial H}{\partial p}\right|_{p=\frac{1-x}{2}} P_{j}+\frac{1+x}{2} P_{j+1}, q=\frac{1-x}{2} Q_{j}+\frac{1+x}{2} Q_{j+1} d x .
\end{aligned}
$$

By taking differentials of the equations (5.2),

$$
\begin{aligned}
d P_{j+1}-d P_{j} & =-\frac{h_{j}}{2} \int_{-1}^{1}\left(H_{q p}\left(\frac{1-x}{2} d P_{j}+\frac{1+x}{2} d P_{j+1}\right)+H_{q q}\left(\frac{1-x}{2} d Q_{j}+\frac{1+x}{2} d Q_{j+1}\right)\right) d x, \\
d Q_{j+1}-d Q_{j} & =\frac{h_{j}}{2} \int_{-1}^{1}\left(H_{p p}\left(\frac{1-x}{2} d P_{j}+\frac{1+x}{2} d P_{j+1}\right)+H_{p q}\left(\frac{1-x}{2} d Q_{j}+\frac{1+x}{2} d Q_{j+1}\right)\right) d x,
\end{aligned}
$$

where $H_{p p}^{\top}=H_{p p}, H_{q q}^{\top}=H_{q q}, H_{p q}^{\top}=H_{q p}$. By taking the wedge product of (5.3) with $d_{j+1}$ and (5.4) with $\mathrm{dP}_{j+1}$, we have

$$
\begin{aligned}
d P_{j+1} \wedge d Q_{j+1}-d P_{j} \wedge d Q_{j+1}= & -\frac{h_{j}}{2} \int_{-1}^{1}\left(H_{q p}\left(\frac{1-x}{2} d P_{j} \wedge d Q_{j+1}+\frac{1+x}{2} d P_{j+1} \wedge d Q_{j+1}\right)\right. \\
& \left.+H_{q q} \frac{1-x}{2} d Q_{j} \wedge d Q_{j+1}\right) d x \\
d Q_{j+1} \wedge d P_{j+1}-d Q_{j} \wedge d P_{j+1}= & \frac{h_{j}}{2} \int_{-1}^{1}\left(H_{p p} \frac{1-x}{2} d P_{j} \wedge d P_{j+1}\right. \\
+ & \left.H_{p q}\left(\frac{1-x}{2} d Q_{j} \wedge d P_{j+1}+\frac{1+x}{2} d Q_{j+1} \wedge d P_{j+1}\right)\right) d x .
\end{aligned}
$$

By subtracting (5.5) from (5.6):

$$
\begin{aligned}
2 d P_{j+1} \wedge d Q_{j+1}= & d P_{j} \wedge d Q_{j+1}-d Q_{j} \wedge d P_{j+1}-\frac{h_{j}}{2} \int_{-1}^{1}\left(H_{q p} \frac{1-x}{2} d P_{j} \wedge d Q_{j+1}\right. \\
& \left.+H_{q q} \frac{1-x}{2} d Q_{j} \wedge d Q_{j+1}+H_{p p} \frac{1-x}{2} d P_{j} \wedge d P_{j+1}+H_{p q} \frac{1-x}{2} d Q_{j} \wedge d P_{j+1}\right) d x .
\end{aligned}
$$


Similarly, by taking wedge product of (5.3) with $\mathrm{dQ}_{j}$, (5.4) with $\mathrm{dP}_{\mathrm{j}}$, then subtract

$$
\begin{aligned}
2 \mathrm{dP}_{j} \wedge \mathrm{dQ}_{j}= & \mathrm{dP}_{j+1} \wedge \mathrm{dQ}_{j}-\mathrm{dQ}_{j+1} \wedge \mathrm{dP}_{j}+\frac{h_{j}}{2} \int_{-1}^{1}\left(\mathrm{H}_{\mathrm{pp}} \frac{1+x}{2} \mathrm{dP}_{j+1} \wedge \mathrm{dP}_{j}\right. \\
& \left.+\mathrm{H}_{\mathrm{pq}} \frac{1+x}{2} \mathrm{dQ}_{j+1} \wedge \mathrm{dP}_{j}+\mathrm{H}_{\mathrm{qp}} \frac{1+x}{2} \mathrm{dP}_{j+1} \wedge d Q_{j}+\mathrm{H}_{\mathrm{qq}} \frac{1+x}{2} \mathrm{dQ}_{j+1} \wedge \mathrm{d} \mathrm{Q}_{j}\right) \mathrm{d} x .
\end{aligned}
$$

By subtracting (5.8) from (5.7) we have

$$
\begin{aligned}
2 d P_{j} \wedge d Q_{j}-2 d P_{j+1} \wedge d Q_{j+1}= & \frac{h_{j}}{2} \int_{-1}^{1} x\left(H_{p p} d P_{j+1} \wedge d P_{j}\right. \\
& \left.+H_{p q} d Q_{j+1} \wedge d P_{j}+H_{q p} d P_{j+1} \wedge d Q_{j}+H_{q q} d Q_{j+1} \wedge d Q_{j}\right) d x .
\end{aligned}
$$

Utilizing (5.3) and properties of wedge product, we obtain

$$
\begin{aligned}
& \frac{h_{j}}{2} \int_{-1}^{1} x H_{p p} d P_{j+1} \wedge d P_{j} d x \\
&= \frac{h_{j}}{2} \int_{-1}^{1} x H_{p p}\left(d P_{j}-\frac{h_{j}}{2} \int_{-1}^{1}\left(H_{q p}\left(\frac{1-x}{2} d P_{j}+\frac{1+x}{2} d P_{j+1}\right)\right.\right. \\
&\left.\left.+H_{q q}\left(\frac{1-x}{2} d Q_{j}+\frac{1+x}{2} d Q_{j+1}\right)\right) d x\right) \wedge d P_{j} d x \\
&=-\frac{h_{j}^{2}}{4}\left(\int_{-1}^{1} x H_{p p} d x \int_{-1}^{1} H_{q p} \frac{1-x}{2} d x d P_{j} \wedge d P_{j}+\int_{-1}^{1} x H_{p p} d x \int_{-1}^{1} H_{q p} \frac{1+x}{2} d x d P_{j+1} \wedge d P_{j}\right. \\
&\left.+\int_{-1}^{1} x H_{p p} d x \int_{-1}^{1} H_{q q} \frac{1-x}{2} d x d Q_{j} \wedge d P_{j}+\int_{-1}^{1} x H_{p p} d x \int_{-1}^{1} H_{q q} \frac{1+x}{2} d x d Q_{j+1} \wedge d P_{j}\right) \\
&= O\left(h^{2}\right) .
\end{aligned}
$$

Similarly, based on (5.9), utilizing (5.3), (5.4), and properties of wedge product, We can prove the following equation

$$
\mathrm{dP}_{\mathrm{j}+1} \wedge d \mathrm{Q}_{j+1}=\mathrm{dP}_{\mathrm{j}} \wedge \mathrm{dQ} \mathrm{Q}_{j}+\mathrm{O}\left(\mathrm{h}^{2}\right) .
$$

We can prove the following theorem.

Theorem 5.1. The linear finite element method of the nonlinear Hamiltonian systems is an approximately symplectic method which have accurate of second to their symplectic condition, i.e., $d p_{j+1} \wedge d q_{j+1}=d p_{j} \wedge d q_{j}+O\left(h^{2}\right)$.

In particular, to linear Hamiltonian systems $H(z)=\frac{1}{2} z^{\top} L z, H_{p p}=A, H_{p q}=C, H_{q q}=B$, then

$$
\int_{-1}^{1} x \mathrm{H}_{p p} \mathrm{dP}_{j+1} \wedge d \mathrm{P}_{j} \mathrm{~d} x=\int_{-1}^{1} x \mathrm{AdP}_{j+1} \wedge \mathrm{dP}_{\mathrm{j}} \mathrm{d} x=0
$$

Based on (5.9), we can prove $d P_{j} \wedge d Q_{j}=d P_{j+1} \wedge d Q_{j+1}$, i.e., the linear finite element methods for the linear Hamiltonian systems is a symplectic algorithm.

\section{Numerical experiments}

Consider nonlinear Hamiltonian system

$$
\mathrm{H}(\mathrm{q}, \mathrm{p})=\mathrm{K}(\mathrm{p})+\mathrm{V}(\mathrm{q})
$$

where $K(p)=2 p_{1}^{2}+p_{2}^{2}$ is the kinetic energy, $V(q)$ is the potential energy,

$$
\mathrm{V}(\mathrm{q})=5 \pi^{2}\left(\mathrm{D}^{2}-5 \mathrm{D}+6.5\right)+4 \mathrm{D}^{-1}+0.5 \pi^{2}\left(\left|\mathrm{q}_{2}\right|-1.5\right)^{2}+\left|\mathrm{q}_{2}\right|^{-1}, \mathrm{D}=\left(\mathrm{q}_{1}^{2}+\mathrm{q}_{2}^{2}\right)^{1 / 2}
$$

The classical Hamiltonian canonical equation is ([4]),

$$
\frac{d p_{1}}{d t}=-\frac{\partial V}{\partial q_{1}}, \frac{d p_{2}}{d t}=-\frac{\partial V}{\partial q_{2}}, \frac{d q_{1}}{d t}=\frac{\partial K}{\partial p_{1}}, \frac{d p_{2}}{d t}=\frac{\partial K}{\partial p_{2}} .
$$

It is the motion of $A_{2} B$ triatomic molecules within the $C_{2 v}$ symmetry. We take the Cartesian coordinate 
system $y \mathrm{O} z$, with origin at the center of mass $\mathrm{O}$, and the $z$ axis is the $\mathrm{C}_{2}$ axis, the coordinates of the two $A$ atoms and $B$ atom is $A\left(y_{1}, z_{1}\right), A\left(y_{2}, z_{2}\right), B\left(y_{3}, z_{3}\right)$. The generalized coordinates $q_{1}=y_{1}-y_{2}, q_{2}=$ $z_{1}-2 z_{3}+z_{2}$, the generalized momentum $p_{1}=0.25 \frac{\mathrm{dq}_{1}}{\mathrm{dt}}, \mathrm{p}_{2}=0.5 \frac{\mathrm{dq}}{\mathrm{dt}}$. Construction of efficient schemes suitable for molecular dynamics applications is an important task. Since very long integration times are required for molecular dynamics simulations, many numerical methods have been developed but the most effective for use in molecular dynamics simulations should have superior long-term stability properties, energy conservation, and permit a large integration time step [1].

We consider the second order symplectic difference scheme (2SS) ([4])

$$
z_{\mathrm{k}+1}=z_{\mathrm{k}}+\mathrm{hJ}^{-1} \mathrm{H}_{z}\left(\frac{z_{\mathrm{k}+1}+z_{\mathrm{k}}}{2}\right) .
$$

The 4-stage, 4th-order explicit symplectic difference scheme (4SS)

$$
\begin{aligned}
H(p, q) & =T(p)+V(q), \alpha=\left(2-2^{\frac{1}{3}}\right)^{-1}, \beta=1-2 \alpha, \\
p_{1} & =p^{n}-c_{1} h\left(\frac{\partial V(q)}{\partial q}\right) q^{n}, q_{1}=q^{n}+d_{1} h\left(\frac{\partial T(p)}{\partial p}\right)_{p_{1},} \\
p_{2} & =p^{n}-c_{2} h\left(\frac{\partial V(q)}{\partial q}\right)_{q_{1},} q_{2}=q^{n}+d_{2} h\left(\frac{\partial T(p)}{\partial p}\right)_{p_{2}}, \\
p_{3} & =p^{n}-c_{3} h\left(\frac{\partial V(q)}{\partial q}\right)_{q_{2}}, q_{3}=q^{n}+d_{3} h\left(\frac{\partial T(p)}{\partial p}\right)_{p_{3}}, \\
p^{n+1} & =p^{n}-c_{4} h\left(\frac{\partial V(q)}{\partial q}\right)_{q_{3}}, q_{n+1}=q^{n}+d_{4} h\left(\frac{\partial T(p)}{\partial p}\right)_{p^{n+1}},
\end{aligned}
$$

where $c_{1}=c_{4}=\frac{\alpha}{2}, c_{2}=c_{3}=\frac{\alpha+\beta}{2}, d_{1}=d_{2}=\alpha, d_{3}=\beta, d_{4}=0$.

Taking initial conditions $\mathrm{q}_{1}(0)=3, \mathrm{q}_{2}(0)=3 / 2, \mathrm{p}_{1}(0)=0, \mathrm{p}_{2}(0)=0$, then energy $\mathrm{H}=50.1951$, stepsize $h=0.01$. Respectively we utilize the linear finite element method (1FEM), 2SS, 4SS, and 4RK to compute the classical trajectories of an $A_{2} B$ type molecule in phase spaces and energy error $\mathrm{H}_{h}-\mathrm{H}$ as follows in Figs 1-7.
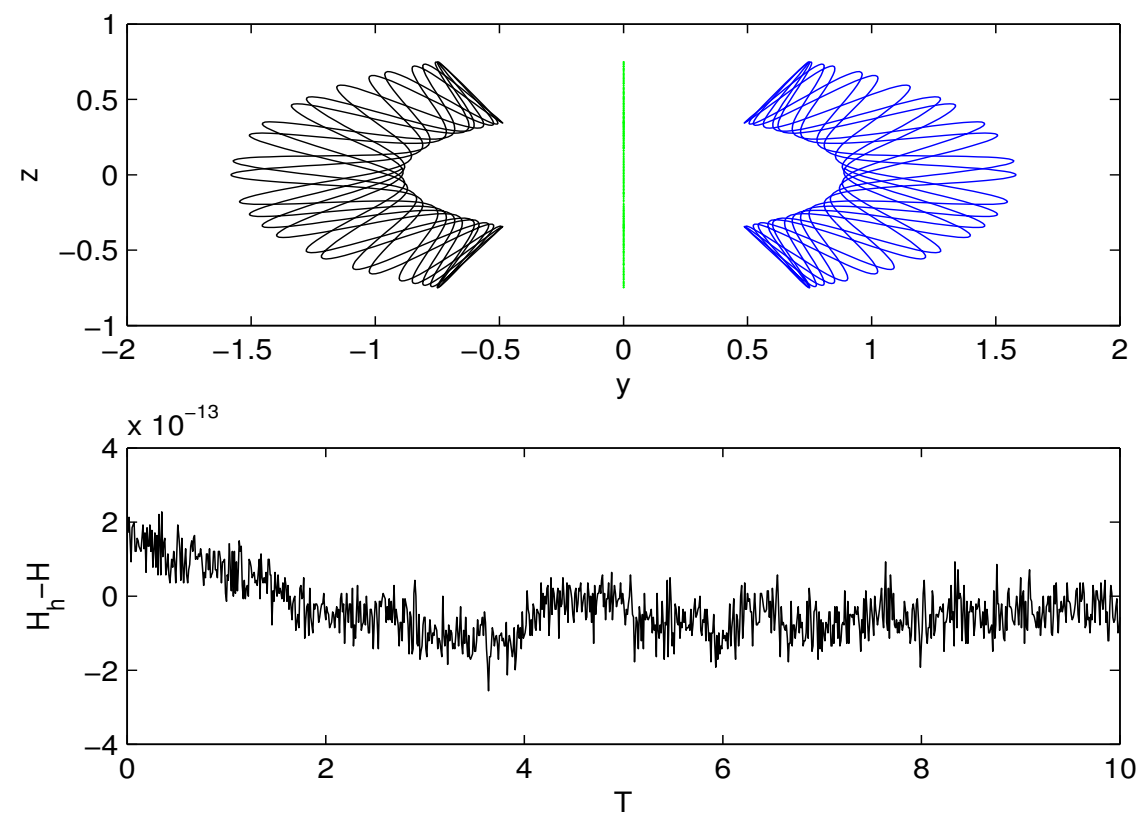

Figure 1: $1 \mathrm{FEM}$, computing $\mathrm{K}=10^{3}$ step, integral interval $\mathrm{T}=[0,10]$, the initial 1000 nodes classical trajectories in phase space and energy error curve. 

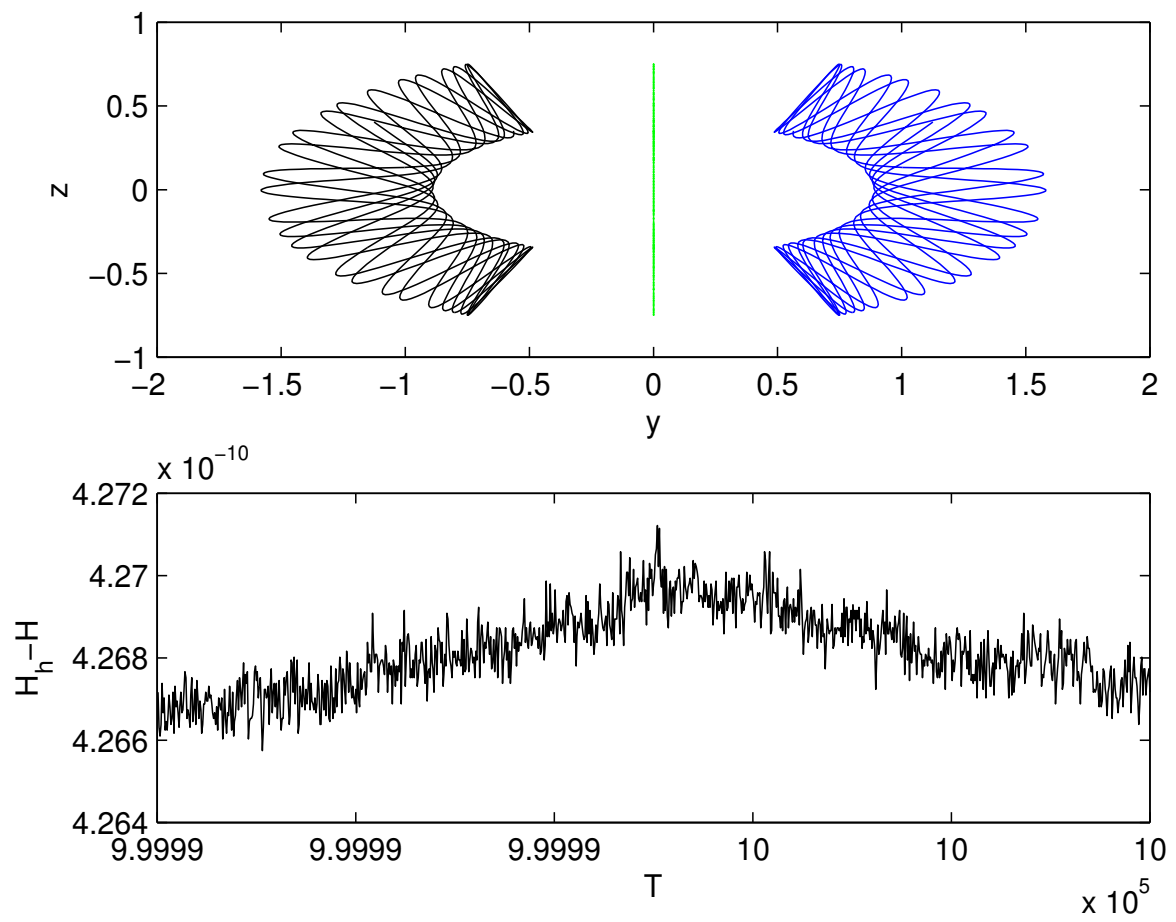

Figure 2: $1 \mathrm{FEM}$, computing $\mathrm{K}=10^{8}$ step, integral interval $\mathrm{T}=\left[0,10^{6}\right]$, the final 1000 nodes classical trajectories in phase space and energy error curve.
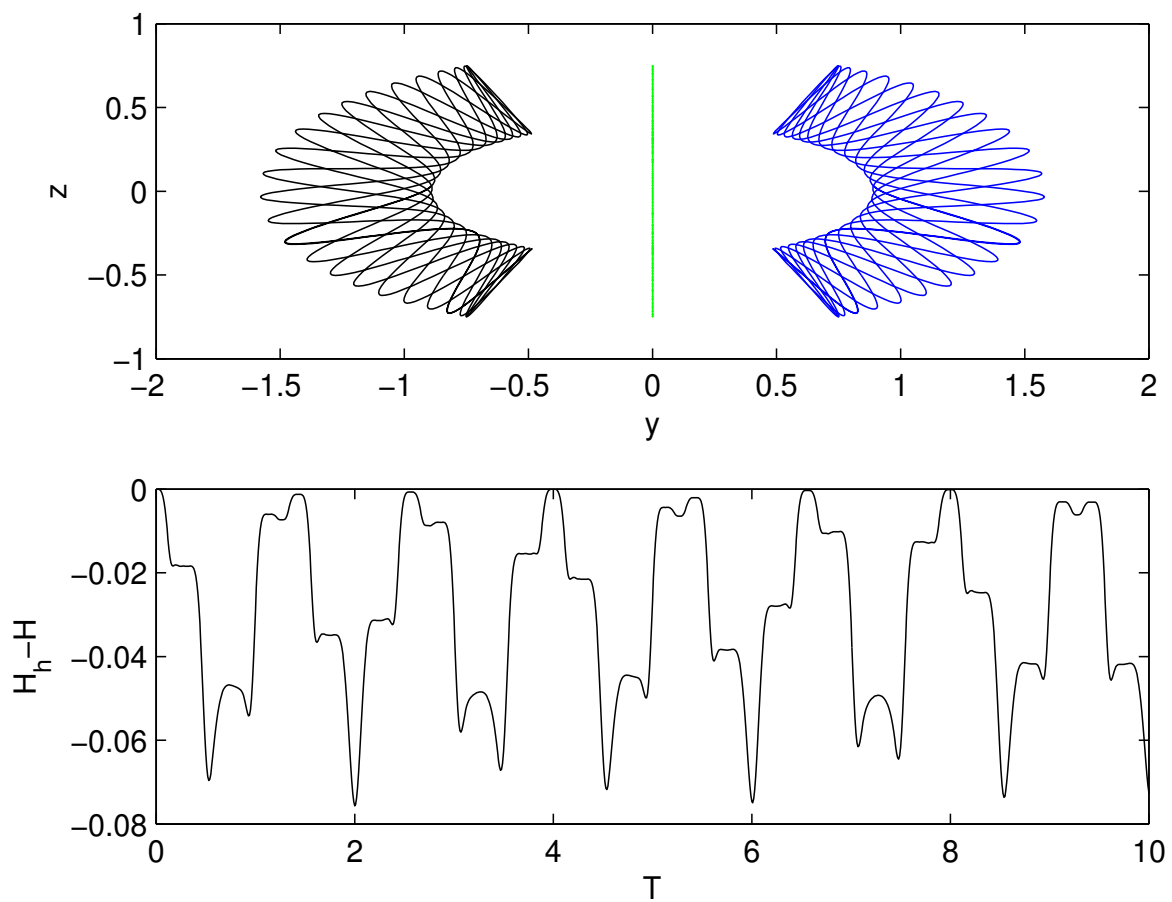

Figure 3: 2SS, computing $\mathrm{K}=10^{3}$ step, integral interval $\mathrm{T}=[0,10]$, the initial 1000 nodes classical trajectories in phase space and energy error curve. 

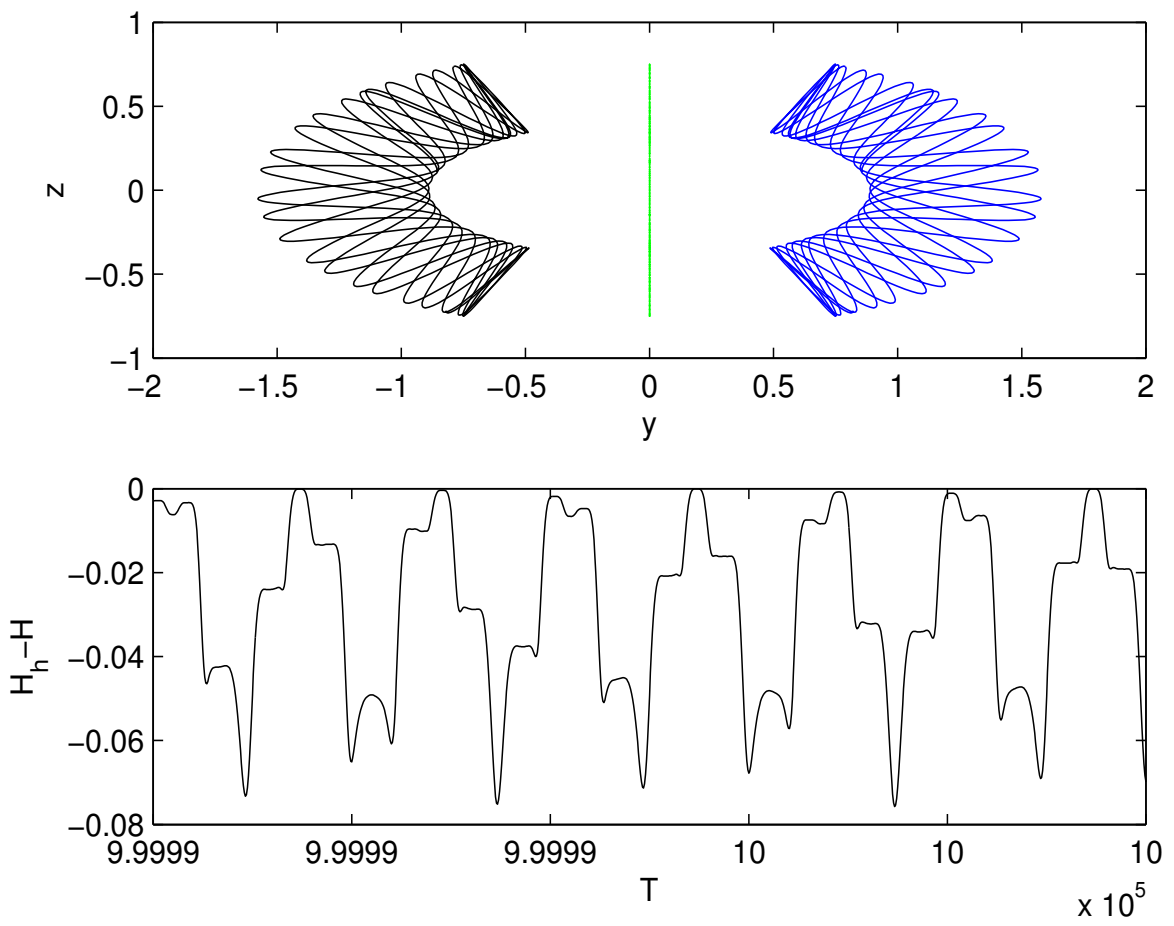

Figure 4: $2 \mathrm{SS}$, computing $\mathrm{K}=10^{8}$ step, integral interval $\mathrm{T}=\left[0,10^{6}\right]$, the final 1000 nodes classical trajectories in phase space and energy error curve.
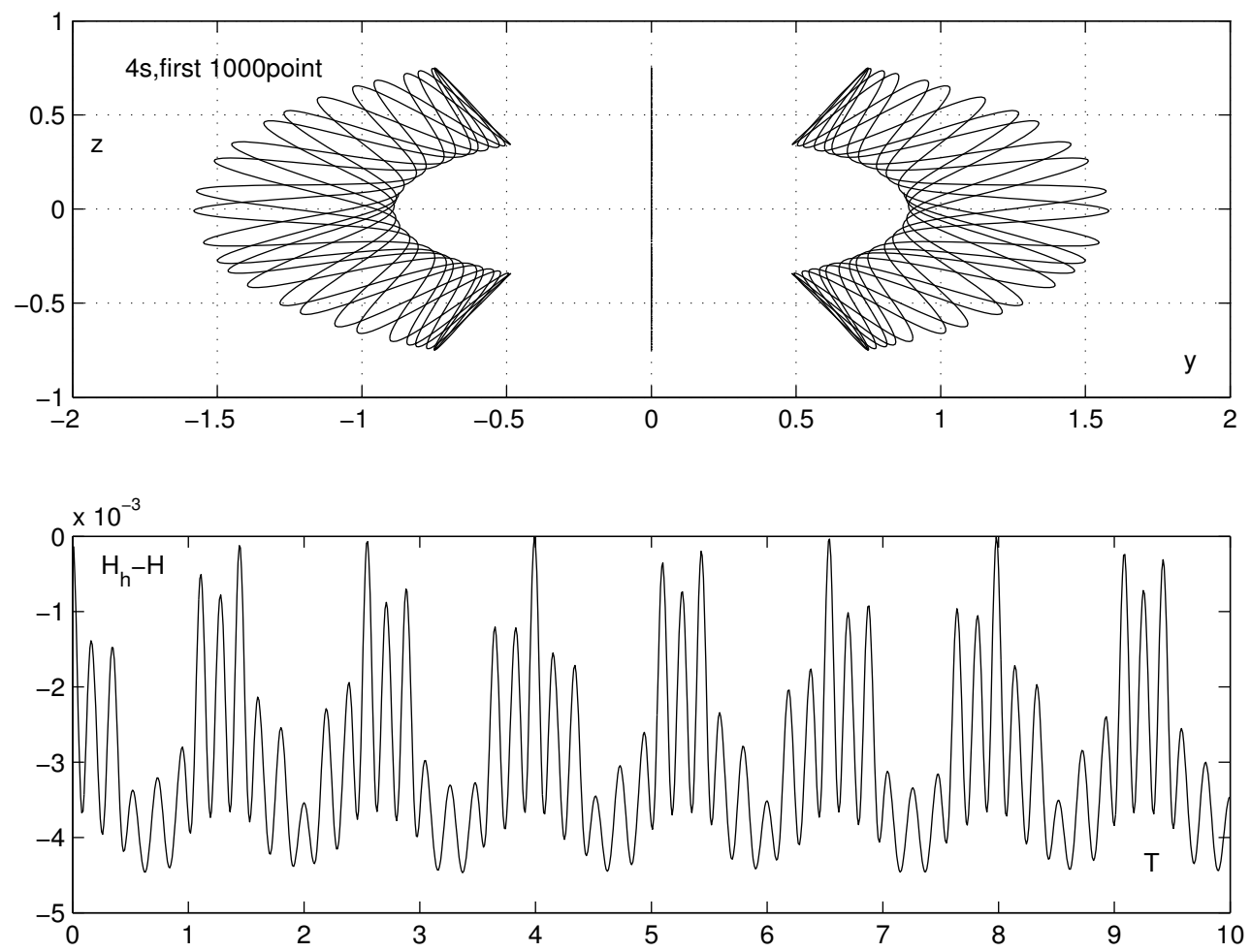

Figure 5: 4SS, computing $\mathrm{K}=10^{3}$ step, integral interval $\mathrm{T}=[0,10]$, the initial 1000 nodes classical trajectories in phase space and energy error curve. 

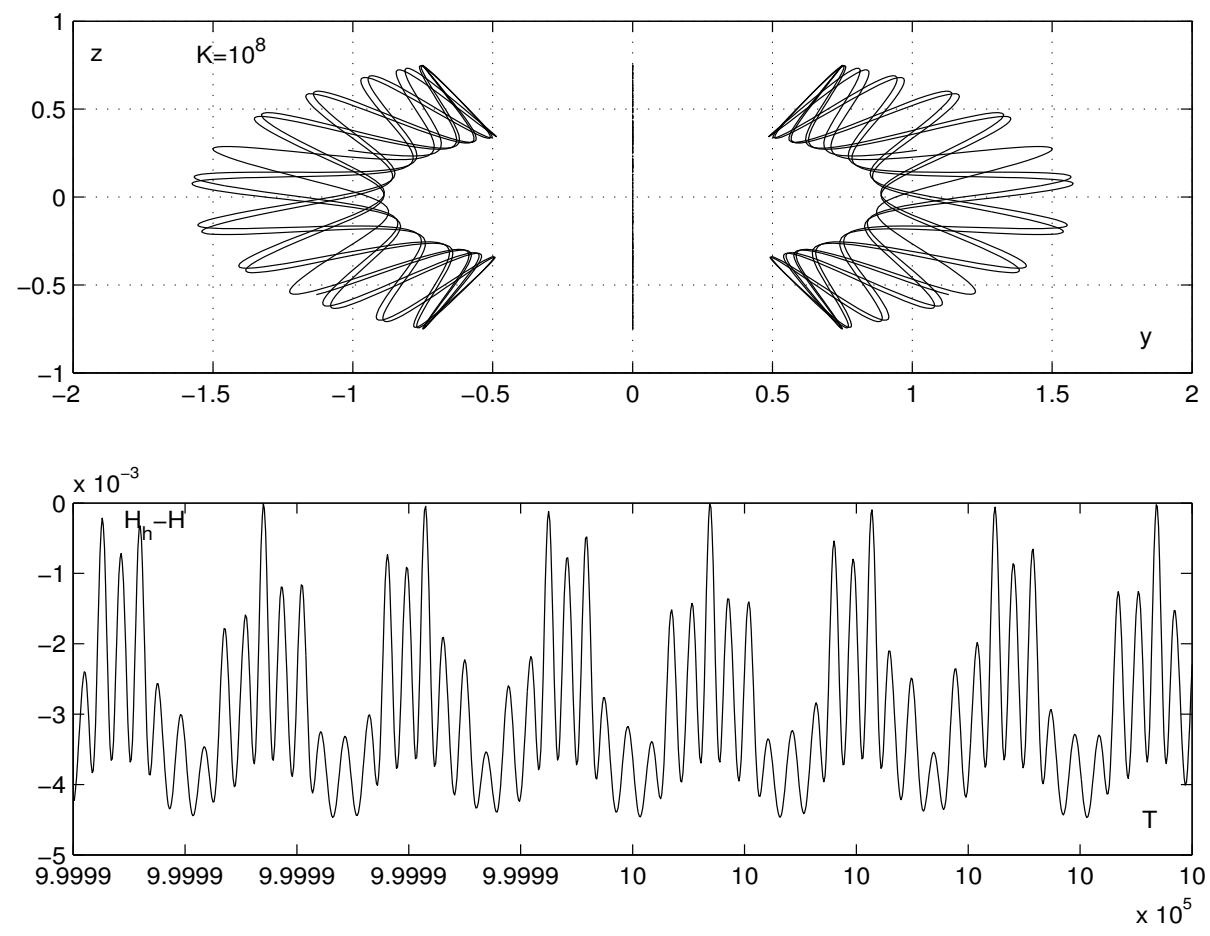

Figure 6: 4SS, computing $\mathrm{K}=10^{8}$ step, integral interval $\mathrm{T}=\left[0,10^{6}\right]$, the final 1000 nodes classical trajectories in phase space and energy error curve.
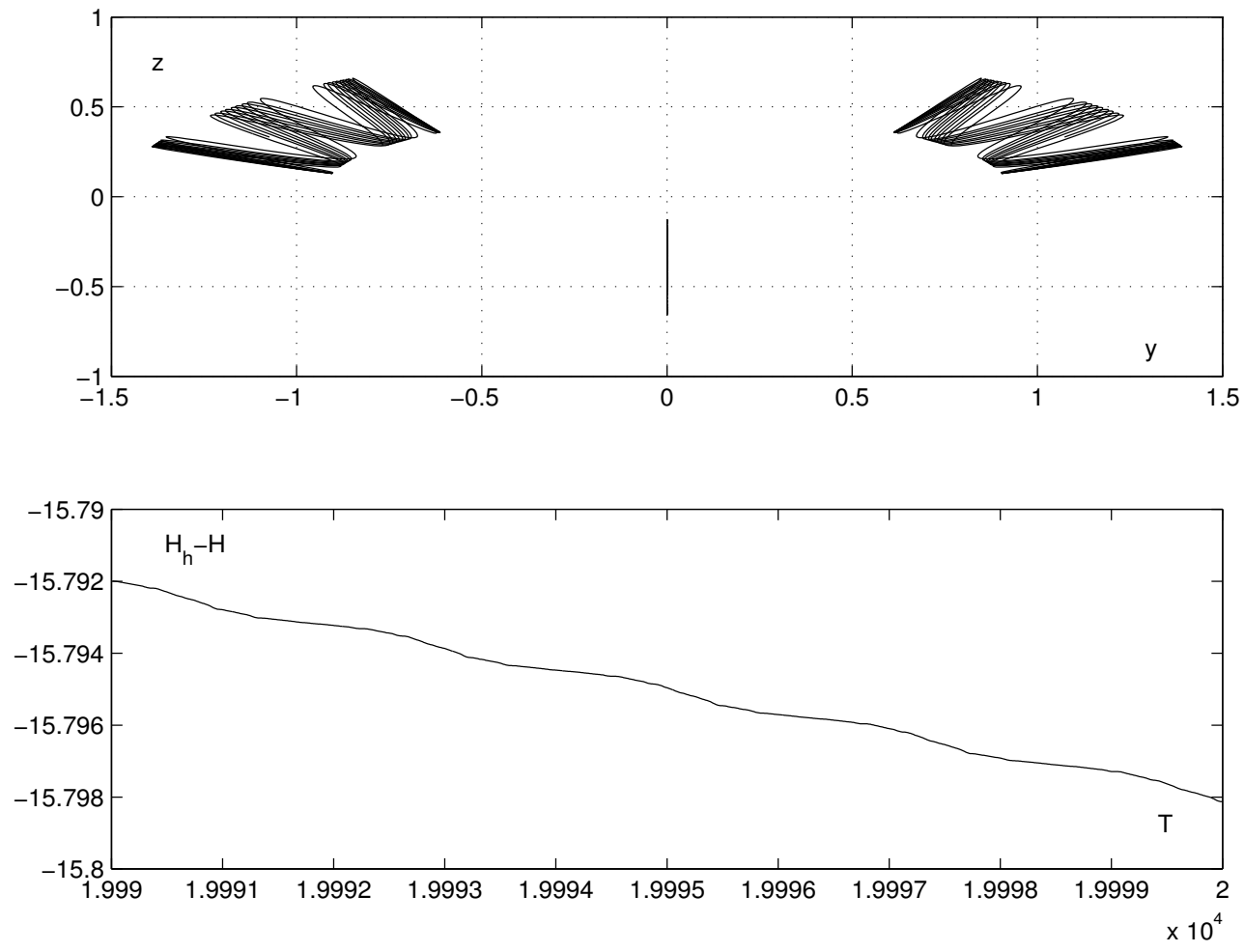

Figure 7: 4RK, computing $\mathrm{K}=2 \times 10^{6}$ step, integral interval $\mathrm{T}=[0,20000]$, the final 1000 nodes classical trajectories in phase space and energy error curve. 
It is observed from Figs 1-7 that the numerical results computed by 1FEM are in good agreement with the theoretical analysis, the atom $B$ and two atoms $A$ in $A_{2} B$ type molecule vibrate quasi-periodically and the phase space are not squeezed together Figs 1 and 2, which indicates that 1FEM can long-time preserve the high accuracy approximation to symplectic structure which just as the symplectic difference method Figs 3-6. However, the numerical results computed by the Runge-Kutta method are not symplectic method, the vibrational range of $A_{2} B$ type molecule shrinks. The energy error computed by the linear element methods is only $10^{-10}$ when $T=1000000$, but the energy deviation is comparatively larger by symplectic difference scheme, energy error up to $10^{-2}(2 \mathrm{SS})$ and $10^{-3}(4 \mathrm{SS})$. It can preserve these basic properties of molecular dynamics.

\section{Conclusion}

The above analysis shows that combing with the wedge product we prove the finite element methods is approximately preserves the symplectic structure to the general Hamiltonian system. It is a reliable method for long time simulations the Hamiltonian system and also provide a better ideas to research the Hamiltonian system.

\section{Acknowledgment}

The authors thank the anonymous reviewers for their useful comments that is helpful to the presentation of this paper. This research was supported by the National Natural Science Foundation of China (No:11101136, 61505054, 11571102), The Science Foundation of Hunan province (No: 2018JJ4062, 14JJ2114), The project of Hunan province department of education(16K026), Science and Technology Innovative Research Team in Higher Educational Institutions of Hunan university, Key Laboratory of Intelligent Information Perception and Processing Technology (Hunan Province).

\section{References}

[1] S. D. Bond, B. J. Leimkuhler, Molecular dynamics and the accuracy of numerically computed averages, Acta Numer., 16 (2007), 1-65. 6

[2] C. M. Chen, Finite element superconvergence construction theory, Hunan Press of Science and Technology, Changsha, (2001). 2

[3] C. M. Chen, Y. Q. Huang, High accuracy theory of finite element, Hunan Press of Science and Technology, Changsha, (1995). 2, 2.1

[4] K. Feng, Collected Works of Feng Kang, National Defence Industry Press, Beijing, (1995). 1, 1.1, 1, 6

[5] K. Feng, M. Z. Qin, Symplectic Geometry Algorithm for Hamiltonian systems, ZheJiang Press of Science and Technology, HangZhou, (2004).

[6] Z. Ge, J. E. Marsden, Lie-Poisson integrators and Lie-Poisson Hamilton-Jacobi theory, Phys. Lett. A, 133 (1988), 134-139. 1

[7] O. Gonzalez, J. C. Simo, On the stability of symplectic and energy-momentum algorithms for nonlinear Hamiltonian systems with symmetry, Comp. Meth. Appl. Mech. Engi., 134 (1996), 197-222.

[8] E. Hairer, C. Lubich, G. Wanner, Geometric Numerical Integration: Structure-Preserving Algorithms for Ordinary Differential Equations, Springer-Verlag Berlin Heidelberg, Berlin, (2006). 1

[9] C. Kane, J. E. Marsden, M. Ortiz, Symplectic-Energy-Momentum Preserving Variational Integrators, J. Math. Phys., 40 (1999), 3353-3371.

[10] F. M. Lasagni, Canonical Runge-Kutta methods, Z. Angew. Math. Phys., 39 (1988), 952-953. 1

[11] B. Leimkuhler, S. Reich, Simulating Hamiltonian Dynamics, Cambridge Universty Press, Cambridge, (2004). 1.2, 1

[12] S. Reich, Multisymplectic Runge-Kutta methods for Hamiltonian wave equation, J. Comput. Phys., 157 (2000), $473-499$.

[13] R. D. Ruth, A canonical intergration technique, IEEE Trans. Nucl. Sci., 30 (1983), 2669-2671.

[14] J. M. Sanz-Serna, Runge-Kutta Schemes for Hamiltonian Systems, BIT, 28 (1988), 877-883. 1

[15] J. M. Sanz-Serna, M. P. Calvo, Numerical Hamiltonian Problems, Chapman \& Hall, London, (1994). 1, 1

[16] A. M. Stuart, A. R. Humphries, Dynamical Systems and Numerical Analysis, Cambridge university press, Cambridge, (1998). 1

[17] Y. B. Suris, The canonicity of mappings generated by Runge-Kutta type methods when integrating the systems $x^{\prime \prime}=-\frac{\partial \mathrm{U}}{\partial x}$, U.S.S.R. Comput. Math. and Math. Phys., 29 (1989), 138-144. 1

[18] Q. Tang, C.-M. Chen, L.-H. Liu, Energy conservation and symplectic properties of continuous finite element methods for Hamiltonian systems, Appl. Math. Comput., 181 (2006), 1357-1368. 1, 2.2 